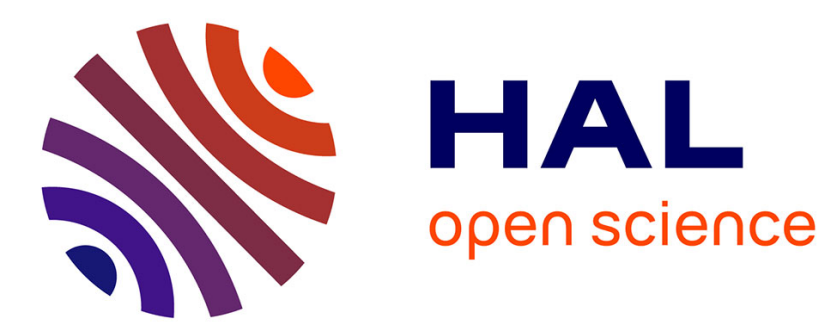

\title{
Microscopic Origins of Shear Strength in Packings Composed of Elongated Particles
}

\author{
Emilien Azéma, Farhang Radjai
}

\section{To cite this version:}

Emilien Azéma, Farhang Radjai. Microscopic Origins of Shear Strength in Packings Composed of Elongated Particles. IWBDG, 2010, France. pp.1-7. hal-00686728

\section{HAL Id: hal-00686728 \\ https://hal.science/hal-00686728}

Submitted on 11 Apr 2012

HAL is a multi-disciplinary open access archive for the deposit and dissemination of scientific research documents, whether they are published or not. The documents may come from teaching and research institutions in France or abroad, or from public or private research centers.
L'archive ouverte pluridisciplinaire HAL, est destinée au dépôt et à la diffusion de documents scientifiques de niveau recherche, publiés ou non, émanant des établissements d'enseignement et de recherche français ou étrangers, des laboratoires publics ou privés. 


\title{
Microscopic origins of shear strength in packings composed of elongated particles
}

\author{
E. Azéma, F. Radjaï
}

\begin{abstract}
We investigate the rheology, force transmission and texture of granular materials composed of elongated particles by means of contact dynamics simulations. The particles have a rounded-cap rectangular (RCR) shape described by a single elongation parameter varying from 0 for a circular particle to 1 for an infinitely thin or long particle. We study the quasi-static behavior, structural and force anisotropies as a function of the elongation parameter for packings submited to biaxial compression. The shear strength is found to increase linearly with this parameter whereas the solid fraction both at the initial isotropic state and in the critical state is nonmonotonous. We show that for these elongated particles a harmonic decomposition of the stress tensor provides a fairly good approximattion of the internal state. Our data suggest that the increase of shear strength with reflects both enhanced friction mobilization and anisotropic particle orientation as the elongation of the particles increases.
\end{abstract}

\section{Introduction}

A wide variety of particle shapes can be found in nature and industry: elongated and platy shapes, angular and facetted shapes, and nonconvex shapes. The issue of shape effect opens actually the door to a vast and substantial scientific domain given a multitude of potential particle morphologies. The effect of particle shape is mediated by the specific granular texture induced by each particle shape. For example, it is found that hard ellipses can be jammed even though they are underconstrained [1]. In the case of anisometric or elongated particle shapes, such as spheroids and sphero-cylinders, the particles tend to develop orientational order affecting force transmission and frictional behavior $[2,3]$. This "nematic" ordering occurs while the particles interact only via contact and friction.

LMGC, CNRS - Université Montpellier 2, Place Eugène Bataillon, 34095 Montpellier cedex 05, France, e-mail: azema@1mgc.univ-montp2.fr ; radjai@1mgc.univ-montp2.fr 
In this paper we focus particularly on the connectivity of particles and we show, depending on the elongation, how each contact type contributes to the internal angle of friction [7]. We first introduce our numerical approach in Section 2. Then, in Section 3, the stress-strain behavior is presented for different values of $\eta$. The microstructure is analyzed in Section 4.

\section{Numerical procedures}

The CD method is based on implicit time integration of the equations of motion and a nonsmooth formulation of mutual exclusion and dry friction between particles $[4,5]$. This method requires no elastic repulsive potential and no smoothing of the Coulomb friction law for the determination of forces. For this reason, the simulations can be performed with large time steps compared to molecular dynamics simulations. We used LMGC90 which is a multipurpose software developed in our laboratory, capable of modeling a collection of deformable or undeformable particles of various shapes by different algorithms [6].

The particles are modeled as a juxtaposition of two half-disks of radius $R^{\prime}$ with one rectangle of length $L$ and width $2 R^{\prime}$ to which we will refer as Rounded-Cap Rectangular (RCR) particles, see Fig. 1 . The RCR shape can be characterized by a single aspect ratio $\alpha$ or by an elongation parameter $\eta=\left(R-R^{\prime}\right) / R=(\alpha-1) / \alpha$ varying from $\eta=0$, for a circle, to 1 corresponding to a line.
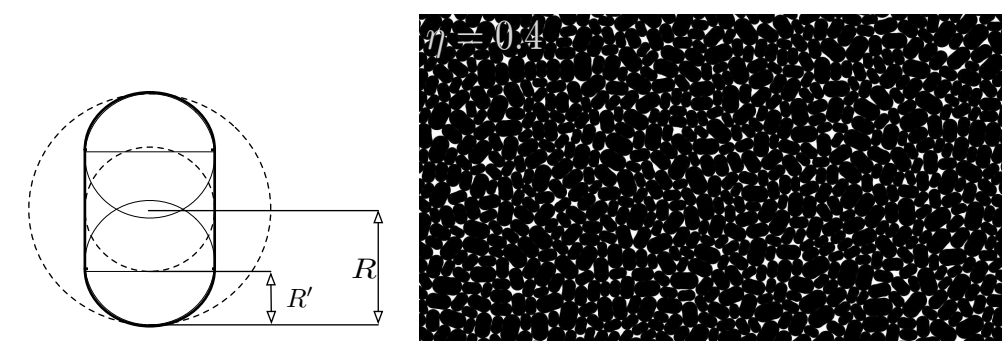

Fig. 1 Shape of a Rounded-Cap Rectangle (RCR) (left). Snapshot of the portion of the packing for $\eta=0.4$ (right).

We prepared 8 different packings of 13000 RCR particles with $\eta$ varying from 0 to 0.7 by steps of 0.1 . The radius $R \in\left[R_{\min }, 3 R_{\min }\right]$ of the circumscribing circle defines the size of a RCR particle. All samples were compacted by isotropic compression inside a rectangular frame of dimensions $l_{0} \times h_{0}$ in which the left and bottom walls are fixed, and the right and top walls are subjected to the same compressive stress $\sigma_{0}$. The gravity was set to zero in order to avoid force gradients in the samples. The coefficient of friction was set to 0 between grains and walls during the isotropic compression. Thus, at equilibrium, all samples were in isotropic stress state. Fig. 1 displays snapshot of the packings for $\eta=0.4$ at the end of isotropic compaction. 
The isotropic samples are then subjected to vertical compression by downward displacement of the top wall at a constant velocity $v_{y}$ for a constant confining stress $\sigma_{0}$ acting on the lateral walls. The friction coefficient $\mu$ between particles is set to 0.5 and to zero with the walls.

\section{Stress-strain behavior}

The stress tensor $\boldsymbol{\sigma}$ can be evaluated from the simulation data as an average over the dyadic product of contact force $f^{c}$ and branch vector $\ell^{c}: \sigma_{\alpha \beta}=n_{c}\left\langle f_{\alpha}^{c} \ell_{\beta}^{c}\right\rangle_{c}$ [4], where $n_{c}$ is the number density of contacts $c$, and the average $\langle\ldots\rangle_{c}$ run over all contacts in a control volume. We can extract the mean stress $p=\left(\sigma_{1}+\sigma_{2}\right) / 2$ as well as the stress deviator $q=\left(\sigma_{1}-\sigma_{2}\right) / 2$ where $\sigma_{1}$ and $\sigma_{2}$ are the principal stresses values. The principal strain values are $\varepsilon_{1}=\int_{h_{0}}^{h} d h^{\prime} / h^{\prime}$ and $\varepsilon_{2}=\int_{l_{0}}^{l} d l^{\prime} / l^{\prime}$. The control variable is the shear strain given by $\varepsilon_{p}=\varepsilon_{1}+\varepsilon_{2}$.
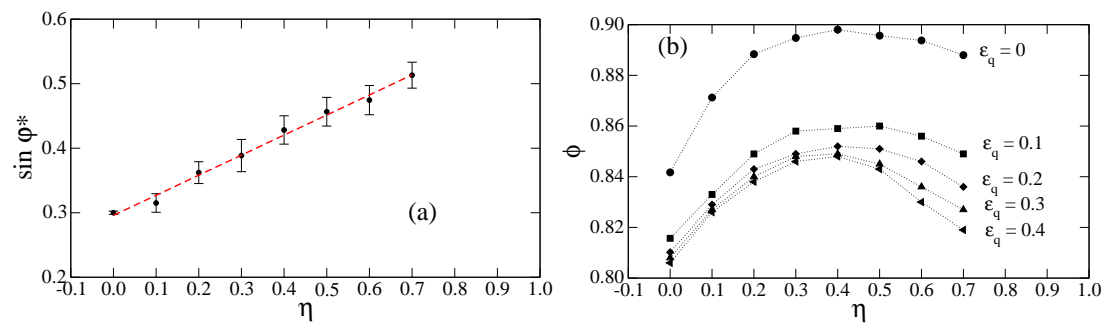

Fig. 2 (a) Internal angle of friction $\varphi^{*}$ as a function elongation $\eta$. (b) Solid fraction as a function of particle shape parameter $\eta$ at different levels of shear strain

During shear, the shear stress jumps initially to a high value before decreasing to a nearly constant value in the steady state. The steady-state shear stress $(q / p)^{*}$ characterizes the shear strength of the material. According to the Mohr-Coulomb model, the internal angle of friction, representing the shear strength of the material, is defined by $\sin \varphi^{*}=(q / p)^{*}$ [8]. Interestingly, as shown by Fig. 2(b), the shear strength varies linearly with the elongation parameter.

Figure 2 displays the solid fraction $\phi$ as a function of $\eta$ at different levels of shear deformation $\varepsilon_{q}$. It is remarkable that, at all levels of deformation, the solid fraction increases with $\eta$, reaches a maximum at $\eta \simeq 0.4$ and then declines as $\eta$ further increases. We note that solid fractions as large as 0.90 are reached for $\eta=0.4$ in the initial state. A similar nonmonotonous behavior was observed for packings of ellipses or ellipsoidal particles [1]. This is somewhat a counterintuitive finding as the shear strength (a monotonous function of $\eta$ ) does not follow the trend of solid fraction (nonmonotonous). 


\section{Granular texture and force transmission}

Remembering that RCR particles are clumps of two disks with one rectangle, a major effect of elongation is then to allow for multiple contacts between two touching particles: cap-to-cap ( $c c)$, cap-to-side ( $c s)$ and side-to-side (ss) contacts in each packing. The side-to-side or side-to-cap contacts are able to accommodate force lines that are usually unsustainable by cap-to-cap contacts. Fig. 3 (left) shows the proportions $k_{c c}, k_{c s}$ and $k_{s s}$ of $c c, c s$ and $s s$ contacts averaged over the residual state as a function of $\eta$. We see that $k_{c c}$ declines with $\eta$ from 1 (for disks) to 0.2 for $\eta=0.7$. At the same time, $k_{c s}$ and $k_{s s}$ increase from 0 to 0.6 and to 0.2 , respectively. Interestingly, $k_{c s} \simeq k_{c c}$ for $\eta \simeq 0.4$, and $k_{s s} \simeq k_{c s}$ for $\eta=0.7$. In this way, as the particle elongation increases, the packing passes from a contact network dominated by $c c$ contacts to a contact network dominated by the complex contacts $c s$ and ss.
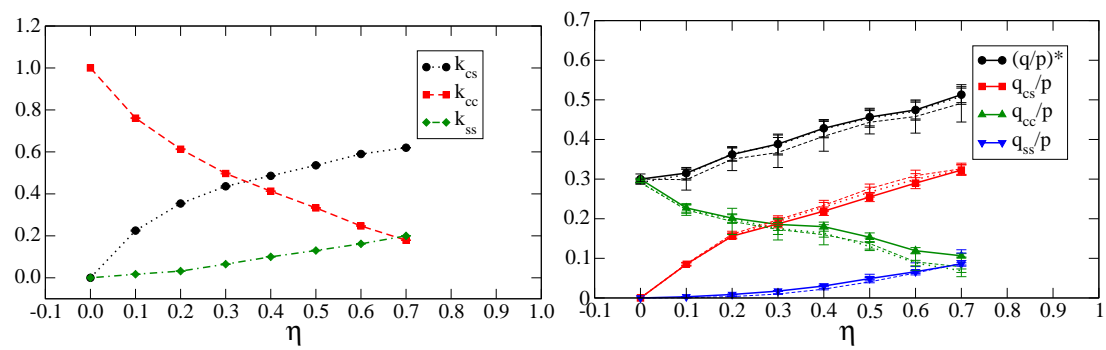

Fig. 3 (left) Proportions of side-to-side ( $s s$ ), cap-to-side $(c s)$ and cap-to-cap ( $c c$ ) contactsand (right) shear strength $(q / p)^{*}$ (circle) for $c s$ (square), ss (triangle up) and $c c$ (triangle down) contacts as a function of $\eta$, together with the harmonic approximation fits (see below).

An additive decomposition of the stress tensor can be performed by grouping the contacts according to their types: $\boldsymbol{\sigma}=\boldsymbol{\sigma}_{c c}+\boldsymbol{\sigma}_{c s}+\boldsymbol{\sigma}_{s s}$, where $\boldsymbol{\sigma}_{c c}, \boldsymbol{\sigma}_{c s}$ and $\boldsymbol{\sigma}_{s s}$ are obtained from the expression of the stress tensor (see sec. 3) by restricting the summation to $c c, c s$ and $s s$ contacts, respectively. The corresponding stress deviators $q_{c c}, q_{c s}$ and $q_{s s}$ are then calculated and normalized by the mean pressure $p$. Note that, by construction we have $q / p=\left(q_{c c}+q_{c s}+q_{s s}\right) / p$. Fig. 3 (right) shows $q_{c c} / p, q_{c s} / p$ and $q_{s s} / p$ averaged in the residual state as a function of $\eta$. We see clearly that $q_{c c} / p$ follows a trend opposite to that of $q_{c s} / p$. For $\eta<0.3,(q / p)^{*}$ is dominated by $c c$ contacts. For $\eta \simeq 0.3, c c$ and $c s$ contacts participate equally to the shear stress, and for $\eta>0.3$, the $c s$ contacts dominate $(q / p)^{*}$. Remarkably, $q_{s s} / p \simeq 0$ for $\eta<0.4$. In this way, the growth of the number of $c s$ and $s s$ contacts is clearly at the origin of a gradual consolidation of the packings as $\eta$ increases.

The shear strength of a granular material reflects its ability to develop force and fabric anisotropies[9, 2, 10, 7]. The fabric anisotropy $a_{c \gamma}$, where $\gamma$ stands alternatively for $\{c c, c s, s s\}$, is the anisotropy of the distribution $P_{\gamma}(\theta)$ of contact orientation $\theta$. At the lowest order, we have 


$$
P_{\gamma}(\theta)=\left\{1+a_{c \gamma} \cos 2\left(\theta-\theta_{\sigma}\right)\right\} / 2 \pi,
$$

where $\theta_{\sigma}$ is the major principal stress direction $\left(\theta_{\sigma}=\pi / 2\right)$. Note that, by construction the whole contact anisotropy is given by $a_{c}=a_{c s s}+a_{c c s}+a_{c c c}$. In the same way, the normal and tangential branch anisotropies $\left(a_{l n}, a_{l t \gamma}\right)$ and forces anisotropies $\left(a_{f n \gamma}, a_{f t \gamma}\right)$ can be defined from the distributions of the angular average of normal and tangential branch length and forces, respectively, by:

$$
\left\{\begin{array}{l}
\left\langle\ell_{n \gamma}\right\rangle(\theta)=\langle\ell\rangle\left\{1+a_{l n \gamma} \cos 2\left(\theta-\theta_{\sigma}\right)\right\} \\
\left\langle\ell_{t \gamma}\right\rangle(\theta)=\langle\ell\rangle a_{l t \gamma} \sin 2\left(\theta-\theta_{\sigma}\right) \\
\left\langle f_{n \gamma}\right\rangle(\theta)=\langle f\rangle\left\{1+a_{f n \gamma} \cos 2\left(\theta-\theta_{\sigma}\right)\right\} \\
\left\langle f_{t \gamma}\right\rangle(\theta)=\langle f\rangle a_{f t \gamma} \sin 2\left(\theta-\theta_{\sigma}\right),
\end{array}\right.
$$

where $\langle f\rangle$ and $\langle\ell\rangle$ are the mean force and the mean branch length. By construction, the total normal and tangential branch and force anisotropies are given by the sum of the corresponding partial anisotropies.

Using the above Fourier approximations together with the expression of the stress tensor it can be shown that $[2,10,7]$ :

$$
\frac{q_{\gamma}}{p} \simeq \frac{1}{2}\left(a_{c \gamma}+a_{l n \gamma}+a_{l t \gamma}+a_{f n \gamma}+a_{f t \gamma}\right)
$$

We can see in Fig.3(right) that this decomposition is nicely verified by our numerical data both for the partial shear stress $q_{\gamma} / p$ as well as for the whole shear stress $(q / p)^{*}$. The contribution of the normal and tangential branch anisotropies is negligible. Fig.4 shows only the partial critical-state anisotropies $a_{c \gamma}, a_{f n \gamma}$, and $a_{f t \gamma}$, together with the total critical-state anisotropies $a_{c}, a_{f n}$ and $a_{f t}$. The anisotropy $a_{c s s}$ of ss contacts increases slowly with $\eta$ from 0 to 0.18 . At the same time, $a_{c c c}$ decreases and at $\eta=0.7$ we have $a_{c s s}=a_{c c c}$. Hence, although the ss contacts represent at $\eta=0.7$ nearly $20 \%$ of contacts, their contribution to the contact anisotropy remains modest and of the same order as $c c$ contacts. The variation of the contact anisotropy $a_{c}$ is thus largely governed by that of $a_{c c s}$. We see also that, the $c s$ contacts carry most of the normal and tangential force anisotropies. The ss contacts contribute modestly to the global force anisotropies only for $\eta \geq 0.4$. The anisotropy $a_{\text {fncc }}$ declines with $\eta$, mainly due to their low number, and $a_{f t c c}$ stays nearly constant. The increase of $a_{c}$ with $\eta$ and a hight value of $a_{f t}$ for the most elongated particle reveal that (1) particles tend to be aligned orthogonally to the compressive direction and (2) a strong mobilization of friction due the increase of the side length of particles $[3,10,7]$.

\section{Conclusions}

In this paper, we applied the contact dynamics method to simulate large samples of elongated particles. It was shown that the shear strength is an increasing lin- 

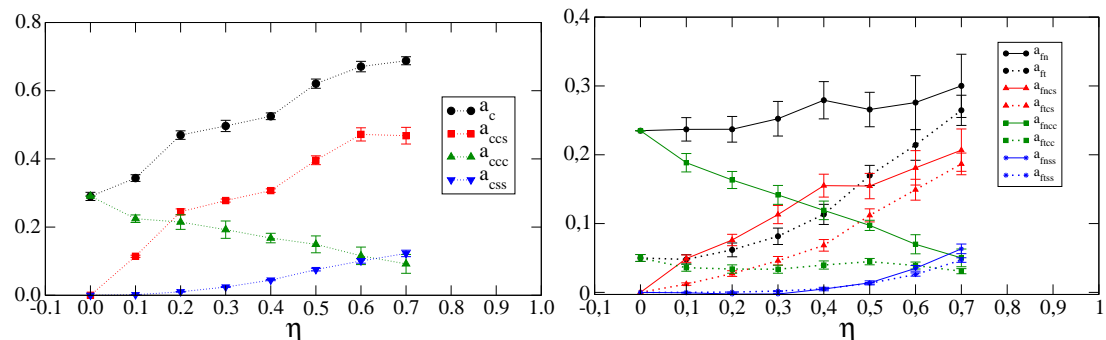

Fig. 4 (left) Partial contact orientation anisotropies $a_{c c c}, a_{c c s}$ and $a_{c s s}$ as a function of $\eta$ in the critical state. (right) Partial normal (line) and tangential (dashed line) force anisotropies $a_{f n c c}$, $a_{f n c s}$ and $a_{f n s s}$, and $a_{f t c c}, a_{f t c s}$ and $a_{f t s s}$ as a function of $\eta$ in the critical state.

ear function of elongation whereas the solid fraction first increases and then declines. We find that both force and texture anisotropies contribute to the increase of shear strength. The increasing mobilization of friction force (and the associated anisotropy) as well as a local nematic ordering of the particles (reveled by contact anisotropy), seem to be the key effect of particle elongation. Currently, we work to extend these results to 3D systems composed with rounded-cap-cylinders (spherocylinders).

\section{References}

1. A. Donev, R. Connelly, F.H Stillinger, S. Torquato, Underconstrained jammed packings of nonspherical hard particles : Ellipses and ellipsoids Phys. Rev. E, 75, 051304 (2007)

2. H. Ouadfel, L. Rothenburg, 'Stress-force-fabric' relationship for assemblies of ellipsoids, Mechanics of Materials, 33, 201-221, (2001).

3. R. C. Hidalgo, I. Zuriguel, D. Maza, I. Pagonabarraga Role of Particle Shape on the Stress Propagation in Granular Packings Phys. Rev. Lett, 103, 118001 (2009)

4. J.J. Moreau Some numerical methods in multibody dynamics : application to granular Eur. J. Mech. A/Solids, 13, 93-114 (1994)

5. F. Radjaï and V. Richefeu Contact dynamics as a nonsmooth discrete element method Mechanics of Materials, 41, 715-728 (2009)

6. F. Dubois, M. Jean The non smooth contact dynamic method: recent LMGC90 software developments and application in Analysis and Simulation of Contact Problems, Springer Berlin / Heidelberg, Volume 27 (2006)

7. E. Azéma, F. Radjai, Stress-strain behavior and geometrical properties of packings of elongated particles, Phys. Rev. E 81, 051304 (2010).

8. J.K. Mitchell, K. Soga, Fundamentals of Soil Behavior (Wiley, New York, 2005)

9. F. Radjai, D. E. Wolf, M. Jean, J.J. Moreau, Bimodal character of stress transmission in granular packings Phys. Rev. Lett, 80, 61-64 (1998)

10. E. Azéma, F. Radjai, R. Peyroux, G. Saussine, Quasistatic rheology, force transmission and fabric properties of a packing of irregular polyhedral particles, Mechanics of Materials, 41, 729-741 (2009) 